\title{
Absolute Spin-Valve Effect with Superconducting Proximity Structures
}

\author{
Daniel Huertas-Hernando, ${ }^{1}$ Yu. V. Nazarov, ${ }^{1}$ and W. Belzig ${ }^{2}$ \\ ${ }^{1}$ Department of Applied Physics and Delft Institute of Microelectronics and Submicrontechnology, \\ Delft University of Technology, Lorentzweg 1, 2628 CJ Delft, The Netherlands \\ ${ }^{2}$ Department of Physics and Astronomy, University of Basel, Klingelbergstrasse 82, 4056 Basel, Switzerland
}

(Received 16 July 2001; published 11 January 2002)

\begin{abstract}
We investigate spin-dependent transport in hybrid superconductor-normal-metal-ferromagnet structures under conditions of the proximity effect. We demonstrate the feasibility of the absolute spin-valve effect for a certain interval of voltages in a system consisting of two coupled trilayer structures. Our results are also valid for noncollinear magnetic configurations of the ferromagnets.
\end{abstract}

DOI: $10.1103 /$ PhysRevLett.88.047003

PACS numbers: 74.50.+r, 72.10.-d, 74.80.Dm

Spin transport in hybrid systems of ferromagnets and normal metals is a very active field of research. This is inspired by prospectives of spin-based electronics or "spintronics" [1]. The feasibility to create and control spin accumulation in such systems by injecting spin polarized current from a ferromagnetic material into a nonmagnetic one is being extensively studied [2]. The theory predicts a variety of novel effects in the case of noncollinear magnetizations [3].

The main attention is given to the so-called spin-valve effect, which provides the mechanism for the giant magnetoresistance (GMR) [4]. An idealized ferromagnetic metal would have electrons with only one direction of spin. The current between two such metals would not go if their magnetizations are opposite. This is the absolute spinvalve effect. The absolute effect is impossible to achieve with common ferromagnetic metals, since electron states of both spin directions are present at the Fermi surface. This is why the actual values of GMR are relatively small. There have been substantial efforts to increase these values by exploring various material combinations [4]. Recent attempts to realize the absolute spin-valve effect concentrated on exotic magnetic materials. A spin polarization up to $80 \%$ was achieved using the dilute magnetic semiconductor $\mathrm{Zn}_{1-x}-\mathrm{Mn}_{x}$ Se [5].

In this Letter we propose a different approach, in which an absolute spin-valve effect can be achieved without using "exotic" compounds. We suggest to use the proximity effect minigap induced in a normal metal by an adjacent superconductor. This minigap has been predicted long ago [6] and has been intensively investigated in recent years [7]. Features related to the proximity effect can be probed by tunneling spectroscopy measurements. The tunneling current between two superconducting proximity structures exhibits a jump at the voltage $e V_{\text {th }}=\left(\tilde{\Delta}_{1}+\tilde{\Delta}_{2}\right), \tilde{\Delta}_{1(2)}$ being the minigaps in the structures. This is a consequence of the sharp peak in the density of states at the minigap edge, which mimics a BCS density of states. The current jump at the threshold voltage is well known for tunneling between superconductors [8].
We use the minigap to achieve an absolute spin-valve effect for the tunneling current between two hybrid structures. Each structure combines a normal metal part with superconducting and magnetic reservoirs, which induce superconducting and magnetic correlations in the normal metal part. The presence of a normal part is essential to provide a physical separation between the sources of superconducting and ferromagnetic correlations. This assures that neither the ferromagnet suppresses superconductivity nor the superconductor affects ferromagnetism. It also provides more control over the strength of the correlations.

We have found that the best result is achieved if the ferromagnet is an insulator. Then the only result of the magnetic correlations is a shift $\pm \tilde{h}$ of the minigap edges for opposite spin directions. The peaks of the density of states are therefore split. If one combines two such structures by a tunnel contact between the normal metal parts, the tunneling current exhibits jumps at different threshold voltages depending on which spin components contribute to the current. In the voltage interval between these threshold voltages, the tunneling current jumps from zero to a finite value differently for parallel than for antiparallel orientations of magnetizations in the two structures. Generally, the results depend on the relative orientation of the magnetizations of the two ferromagnets in the system, as well as on the induced superconducting gaps and the induced spin splitting in each normal metal.

A possible design for an actual device is shown in Fig. 1. It consists of two superconductor-normal-metalferromagnet $(S-N-F)$ structures as described above with their normal parts connected by a tunnel junction. For the calculation, we adopt the circuit theory description of the system [9]. In terms of Green's functions, this means that we assume isotropic Green's functions in momentum space. Quasiclassical Green's functions methods have already been used to study structures involving superconducting materials and magnetically active interfaces [10]. The advantage of the circuit theory description is that we do not have to specify a concrete geometry of the structures. Each part of the structure is then presented by a 


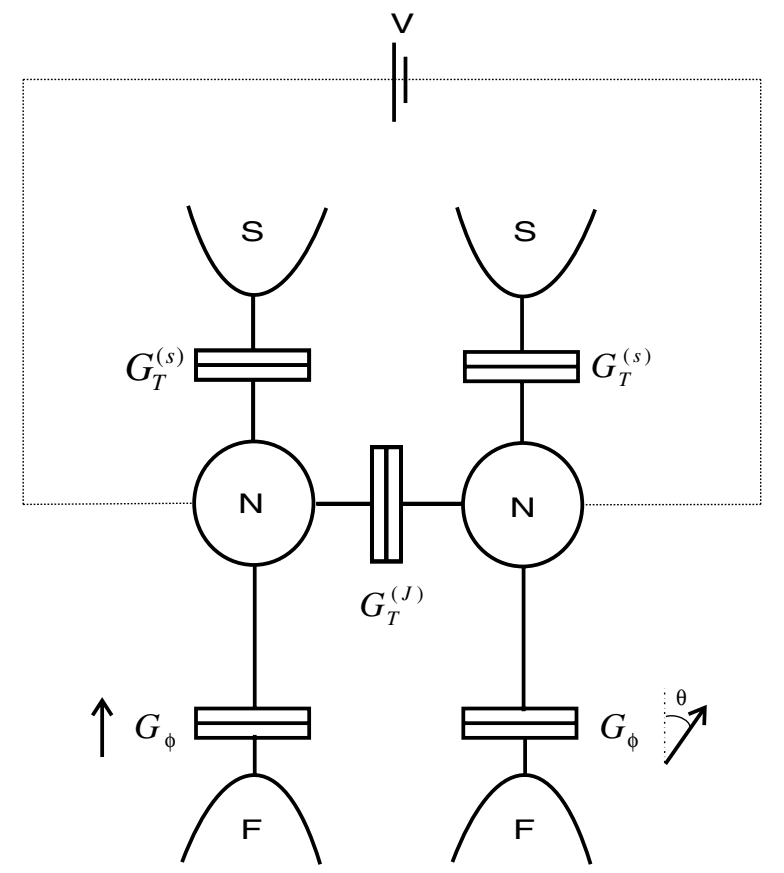

FIG. 1. Schematic circuit of two coupled trilayer $S-N-F$ structures. In each trilayer structure, a normal metal node $(N)$ is coupled to superconducting $(S)$ and ferromagnetic $(F)$ reservoirs through tunnel junctions of conductances $G_{\mathrm{T}}^{(\mathrm{S})}$ and $G_{\phi}$, respectively. The ferromagnetic reservoir is assumed to be a magnetic insulator. Both normal metal nodes are coupled together through a third tunnel junction $G_{\mathrm{T}}^{(\mathrm{J})}$. The relative magnetic configuration of the ferromagnetic insulators may be noncollinear. A voltage $V$ is applied between both $N$ nodes.

normal node, which is connected to superconducting and ferromagnetic reservoirs by means of tunnel junctions. We concentrate first on one of the structures.

In the circuit theory, the Green's functions are calculated from balance equations for matrix "currents" in each node. These currents come from each connector to the node. The matrix current is expressed in terms of the connector properties and the Green's functions on the two sides of the connector. The case of a matrix current, which accounts for both the ferromagnetic and the superconducting nature of the reservoirs, as well as for the magnetic structure of the contact, has not yet been included into the circuit theory. We have investigated this problem in some details [11]. Here, we give only the results for the relevant case of a tunnel connector:

$$
\begin{aligned}
\check{I}_{21}= & \frac{G_{\mathrm{T}}}{2}\left[\check{G}_{2}, \check{G}_{1}\right]+\frac{G_{\mathrm{MR}}}{4}\left[\left\{\vec{M} \hat{\vec{\sigma}}_{\tau_{3}}, \check{G}_{2}\right\}, \check{G}_{1}\right] \\
& +i \frac{G_{\phi}}{2}\left[\vec{M} \hat{\vec{\sigma}} \hat{\tau}_{3}, \check{G}_{1}\right] .
\end{aligned}
$$

Here $\check{G}_{1(2)}$ are the Green's functions on the two sides of the junction. They are matrices in Keldysh-Nambu-spin space, obeying the normalization condition $\breve{G}^{2}=\breve{1}$ [12]. The first term presents the usual boundary condition for tunnel junctions [9], $G_{\mathrm{T}}$ being the junction conductance. The second term accounts for the different conductances for different spin directions. This term leads to a spin polarized current through the junction. We assume a small value of this effect, $G_{\mathrm{MR}} \sim G_{\mathrm{T}}^{\uparrow}-G_{\mathrm{T}}^{\downarrow} \ll G_{\mathrm{T}}$. The unit vector $\vec{M}$ is in the direction of the magnetization, and $\hat{\vec{\sigma}}, \hat{\tau}$ are Pauli matrices in spin and Nambu space, respectively.

The third term is of the most interest for us. It will not vanish even if there is no conductance through the junction. In this special case, the physical meaning of the third term can be understood as follows: electrons with different spin directions pick up different phases when reflecting from the magnetic insulator. The coefficient $G_{\phi}$ is related to the mixing conductance introduced in [3] via $G_{\phi}=\operatorname{Im} G^{\uparrow \downarrow}$. To give a concrete example, we have calculated $G_{\phi}$ in the framework of an effective mass model for electrons with Fermi momentum $k_{\mathrm{f}}$ and with spin-dependent penetration depths $\kappa_{\uparrow, \downarrow}^{-1}$ [13]. Assuming $\delta \kappa \equiv \kappa_{\uparrow}-\kappa_{\downarrow} \ll \kappa$, we find $G_{\phi}=16 A G_{\mathrm{Q}} \kappa \delta \kappa \arcsin \left(i k_{\mathrm{f}} / \kappa\right) /\left(k_{\mathrm{f}}^{2}+\kappa^{2}\right), A$ being the surface area of the interface and $G_{\mathrm{Q}} \equiv e^{2} / 2 \pi \hbar$. First principles calculations of these interface spin conductances have been performed recently [14].

We proceed by finding the Green's functions for equilibrium conditions. In particular, it is sufficient to find the solution in the retarded block only. The retarded Green's functions associated with the ferromagnetic and with the superconducting reservoirs are, respectively, $\hat{R}_{\mathrm{F}}=\hat{\tau}_{3}$ and $\hat{R}_{\mathrm{S}}=\hat{\tau}_{1}$, assuming that the range of energies considered is smaller than the gap of the superconducting reservoir $\left(\varepsilon \ll \Delta_{\text {bulk }}\right)$. The retarded function $\hat{R}$ in the normal metal is obtained from the conservation of matrix currents in the node. The current from the superconductor is given by the first term in (1), and the current from the ferromagnetic insulator is given by the third term. A further current (called "leakage current" in Ref. [9]), being proportional to energy $\varepsilon$ and inversely proportional to the average level spacing $\delta$ in the normal node, is also included. It describes decoherence between electrons and holes. The matrix current conservation then reads

$$
\left[-i G_{\mathrm{Q}} \frac{\varepsilon}{\delta} \hat{\tau}_{3}-i \frac{G_{\phi}}{2} \vec{M} \hat{\vec{\sigma}} \hat{\tau}_{3}+\frac{G_{\mathrm{T}}^{(\mathrm{S})}}{2} \hat{\tau}_{1}, \hat{R}\right]=0,
$$

where $G_{\mathrm{T}}^{(\mathrm{S})}$ is the conductance of the tunnel junction to the superconductor. This equation is easy to solve since it again separates into two blocks for spin parallel $(\uparrow)$ and antiparallel $(\downarrow)$ to the magnetization. We introduce parameters $\tilde{h} \equiv G_{\phi} \delta / 2 G_{\mathrm{Q}}$ and $\tilde{\Delta}=G_{\mathrm{T}}^{(\mathrm{S})} \delta / 2 G_{\mathrm{Q}}$. In these notations, the normalized density of states in the normal node is different for two spin directions and reads

$$
v^{\dagger(\downarrow)}(\varepsilon)=\frac{|\varepsilon \pm \tilde{h}|}{\sqrt{(\varepsilon \pm \tilde{h})^{2}-\tilde{\Delta}^{2}}} .
$$

This expression is the same as the one for a BCS superconductor in the presence of the spin-splitting magnetic field [15]. However, here the density of states is formed in the normal metal, where neither superconductivity nor magnetization is present. The quantities $\tilde{\Delta}, \tilde{h}$ are induced 
by the corresponding reservoirs. This is why superconductivity and ferromagnetism do not have to compete and the relevant parameters can be experimentally controlled by adjusting the conductivities of the barriers [16].

Having obtained the simple solution (3), we discuss now the limits of its validity. The first limitation is the presence of sufficiently strong scattering in the normal part and/or at its boundaries to provide the isotropy of the Green function. Two other limitations are provided by the homogeneity of the Green's function in the node. The minimum size $L$ of the normal part should exceed neither the superconducting coherence length nor the spin-flip length. If the size of the system is larger than the spin-flip length, the circuit theory description fails and spatially dependent Green's functions have to be considered. In addition, the conductance of the normal part itself should exceed both $G_{\phi}$ and $G_{\mathrm{T}}^{(\mathrm{S})}$.

Now we consider transport between the two $S-N-F$ structures through a nonmagnetic tunnel junction with conductance $G_{\mathrm{T}}^{(\mathrm{J})}$ connecting the two normal metals (see Fig. 1). Both structures are assumed to be in local equilibrium. This assumption is justified if $G_{\mathrm{T}}^{(\mathrm{J})} \ll G_{\phi}$, $G_{\mathrm{T}}^{(\mathrm{S})}$. A voltage $V$ is applied between them. We also assume that the temperature $T$ is much smaller than $\tilde{\Delta}$, $\tilde{h}$. This is required for the absolute spin-valve effect. The magnetization directions $\vec{M}_{1(2)}$ of each magnetic insulator may be arbitrary. The matrix current between the two nodes reads

$$
\check{I}=\frac{G_{\mathrm{T}}^{(\mathrm{J})}}{2}\left[\check{G}_{1}, \check{G}_{2}\right]
$$

where $\breve{G}_{1(2)}$ are the quasiclassical Green's functions for the left (1) and for the right node (2), respectively. We can choose the spin-quantization axis to be parallel to $\vec{M}_{1}$. As a result, the Green's function $\breve{G}_{1}$ separates into two blocks in spin space

$$
\check{G}_{1}=\left[\begin{array}{cc}
\check{G}_{1}^{\Uparrow} & 0 \\
0 & \check{G}_{1}^{\Downarrow}
\end{array}\right] .
$$

The Green's function $\check{G}_{2}$ can be presented as

$$
\check{G}_{2}=U\left[\begin{array}{cc}
\check{G}_{2}^{\Uparrow} & 0 \\
0 & \check{G}_{2}^{\Downarrow}
\end{array}\right] U^{-1} .
$$

where $U$ is the spin rotation matrix that transforms $\vec{M}_{2}$ into $\vec{M}_{1}$. The electric current is given by the Keldysh component of Eq. (4):

$$
I_{e}=-\frac{G_{\mathrm{T}}^{(\mathrm{J})}}{8 e} \int_{-\infty}^{\infty} d \varepsilon \operatorname{Tr}\left\{\hat{\tau}_{3}\left[\check{G}_{1}, \check{G}_{2}\right]^{\mathrm{K}}\right\} .
$$

It may be written as

$$
I_{\mathrm{e}}=\frac{1}{4} I_{\mathrm{p}}+\frac{1}{4} I_{\theta}\left(\vec{M}_{1} \cdot \vec{M}_{2}\right),
$$

where $I_{\mathrm{p}, \theta}=I^{\Uparrow}+I^{\Downarrow \downarrow} \pm I^{\Uparrow \downarrow} \pm I^{\downarrow \uparrow}$. Each $I^{s s^{\prime}}\left(s\right.$ and $s^{\prime}=$ $\{\uparrow, \downarrow\})$ is an integral of the form [17]

$$
I^{s s^{\prime}}=\frac{G_{\mathrm{T}}^{(\mathrm{J})}}{e} \int_{0}^{e V} d \varepsilon v_{1}^{s}(\varepsilon-e V) v_{2}^{s^{\prime}}(\varepsilon)
$$

As a function of the applied bias voltage, the left density of states $v_{1}^{s}(\varepsilon-e V)$ is shifted in energy. Now we assume $\tilde{\Delta}_{1(2)} \geqslant \tilde{h}_{1(2)}$. Each component $I^{s s^{\prime}}$ will be zero until the voltage reaches a certain threshold $e V_{\mathrm{th}}^{s s^{\prime}}$, at which both left and right densities of states start to overlap. Because both densities of states are spin split, there are four different threshold voltages $e V_{\mathrm{th}}^{s s^{\prime}}$, depending on which spin components of both densities of states are "matched" together:

$$
e V_{\mathrm{th}}^{s s^{\prime}}=\tilde{\Delta}_{1}+\tilde{\Delta}_{2} \mp\left(\tilde{h}_{1} \pm \tilde{h}_{2}\right) .
$$

So the voltage interval $\left|e V-\tilde{\Delta}_{1}-\tilde{\Delta}_{2}\right| \leqslant \tilde{h}_{1}+\tilde{h}_{2}$ can be divided in four regions, separated by the four different threshold voltages $e V_{\mathrm{th}}^{s s^{\prime}}$.

To illustrate the effect, we consider the symmetric case $\tilde{\Delta}_{1}=\tilde{\Delta}_{2} \equiv \tilde{\Delta}, \tilde{h}_{1}=\tilde{h}_{2} \equiv \tilde{h}$. In this case, there are only three threshold voltages $e V_{\text {th }}^{\downarrow \uparrow}=2(\tilde{\Delta}-\tilde{h}), e V_{\text {th }}^{\Uparrow \uparrow(\downarrow)}=2 \tilde{\Delta}$, and $e V_{\text {th }}^{\uparrow \downarrow}=2(\tilde{\Delta}+\tilde{h})$. At each threshold, the correspondent spin component $I^{s s^{\prime}}$ jumps from zero to the value

$$
I^{s s^{\prime}} \approx \frac{\pi}{4} \frac{G_{\mathrm{T}}^{(\mathrm{J})}}{e} e V_{\mathrm{th}}^{s s^{\prime}} .
$$

These jumps are characteristic of tunneling between superconductors ( $S-S$ tunneling) [8]. Through the voltage interval $|e V-2 \tilde{\Delta}| \leqslant 2 \tilde{h}$, the total current $I_{\mathrm{e}}$ presents steps reflecting these jumps (Fig. 2). These steps depend on the relative angle $\theta$ between the magnetization of the magnetic insulators (see Fig. 2). Of specific interest is the first jump of the current in antiparallel configuration $(\theta=\pi)$, occurring at the threshold $e V_{\mathrm{th}}^{\downarrow \uparrow}=2(\tilde{\Delta}-\tilde{h})$. In this case only spin-down quasiparticles in the left node overlap with spin-up quasiparticles in the right node, which constitutes the absolute spin-valve effect. As expected, the total current, being finite at $\theta=\pi$, goes to zero if the magnetization of one of the ferromagnetic insulators is reversed (see Fig. 2). The absolute spin-valve effect already vanishes at the second zone. Nevertheless again the difference between $\theta=\pi$ and $\theta=0$ currents resembles the effect. Generally these results depend on the relative values of $\tilde{h}_{1(2)}$ and $\tilde{\Delta}_{1(2)}$. In general, the region of voltages where the effect occurs $e V_{\text {th }}^{\Downarrow \downarrow}-e V_{\text {th }}^{\downarrow \uparrow}$ is equal to $2 \min \left(\tilde{h}_{1}, \tilde{h}_{2}\right)$.

In conclusion, we have investigated theoretically spin transport in multiterminal $S-N-F$ proximity structures using quasiclassical Green's function methods, inspired by circuit theories of mesoscopic transport [3,9]. Spin splitting of the induced density of states, caused by the presence of magnetic insulators, is probed by means of tunneling spectroscopy of the superconducting proximity effect. The tunneling current has jumps for certain intervals of voltages, in which an absolute spin-valve effect can be achieved. These features of the current depend on the relative angular configuration of the different magnetic insulators and on the relative values of the 


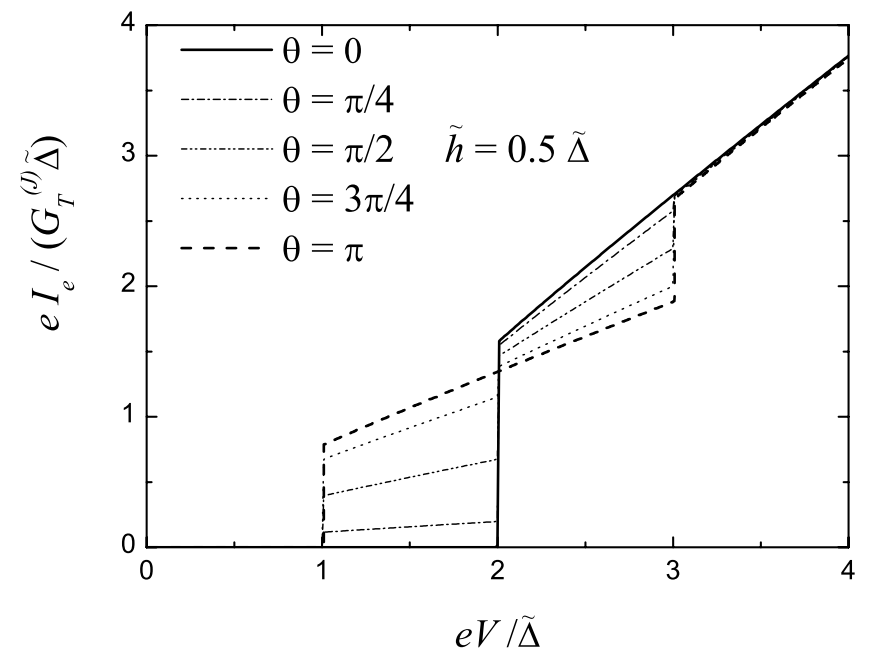

FIG. 2. Steps of the normalized $N-N$ tunneling current $e I_{\mathrm{e}} /\left(G_{\mathrm{T}}^{(\mathrm{J})} \tilde{\Delta}\right)$ with the applied voltage $V$ for the symmetric case $\tilde{\Delta}_{1}=\tilde{\Delta}_{2} \equiv \tilde{\Delta}, \tilde{h}_{1}=\tilde{h}_{2} \equiv \tilde{h}$. In this case $\tilde{h} / \tilde{\Delta}=0.5$. The tunneling current presents jumps in the range of voltages $\mid e V-$ $2 \tilde{\Delta} \mid \leqslant 2 \tilde{h}$. For $\theta=0$, the current jumps at the voltage $e V=$ $2 \tilde{\Delta}$. For $\theta=\pi$ the current presents two jumps at voltages $e V=2(\tilde{\Delta}-\tilde{h})$ and $e V=2(\tilde{\Delta}+\tilde{h})$, respectively. These jumps reflect how the different spin components of the induced density of states in each normal node contribute to the total tunneling current at different voltages. Between the voltages $e V=2(\tilde{\Delta}-\tilde{h})$ and $e V=2 \tilde{\Delta}$ the absolute spin-valve effect is achieved. The change of the current between $\theta=0$ and $\theta=\pi$ situations is shown for various values of the angle $\theta$.

induced superconducting minigap and the induced spin splitting in each node. Moreover, our proposal allows for the possibility of inducing two independent "fields" (i.e., antiparallel fields) in the device. This is very difficult to achieve with an applied magnetic field in a system of superconducting electrodes. Finally, we emphasize that the physical separation of the sources of both superconducting and ferromagnetic correlations provides a feasible way to manipulate specifically the spin-filtering properties of our proposed multiterminal $S-N-F$ proximity structure.

We thank A. Brataas, Ya. M. Blanter, Gerrit E. W. Bauer, and M.R. Wegewijs for discussions. We specially thank N. M. Chtchelkatchev for stimulating discussions and for his help with the numerical calculations. This work was financially supported by the Stichting voor Fundamenteel Onderzoek der Materie (FOM).
[1] G. A. Prinz, Science 282, 1660-1663 (1998); S. Datta and B. B. Das, Appl. Phys. Lett. 56, 665 (1990); J. M. Kikkawa and D. D. Awschalom, Nature (London) 397, 139 (1999); R. Fiederling et al., Nature (London) 402, 787 (2000); Y. Ohno et al., Nature (London) 402, 790 (2000); I. Malajovich et al., Phys. Rev. Lett. 84, 1015 (2000).

[2] F. J. Jedema et al., Nature (London) 410, 345 (2000); A. Filip et al., Phys. Rev. B 62, 9996 (2000).

[3] A. Brataas et al., Phys. Rev. Lett. 84, 2481 (2000); D. Huertas-Hernando et al., Phys. Rev. B 62, 5700 (2000).

[4] M. A. M. Gijs and G.E.W. Bauer, Adv. Phys. 46, 285 (1997).

[5] Th. Gruber, Appl. Phys. Lett. 78, 1101 (2001).

[6] W. L. McMillan, Phys. Rev. 175, 537 (1968).

[7] S. M. Freake and C. J. Adkins, Phys. Lett. 7, 382 (1969); J. Vrba and S. B. Woods, Phys. Rev. B 3, 2243 (1971); J. R. Toplicar and D. K. Finnemore, Phys. Rev. B 16, 2072 (1977); S. Guéron et al., Phys. Rev. Lett. 77, 3025 (1996); W. Belzig et al., Phys. Rev. B 54, 9443 (1996); E. Scheer et al., Phys. Rev. Lett. 86, 284 (2001); N. Moussy et al., Europhys. Lett. 55, 861 (2001); M. Zareyan et al., Phys. Rev. Lett. 86, 308 (2001).

[8] W. L. McMillan and J. R. Rowell, in Superconductivity, edited by R. D. Parks (Marcel Dekker, New York, 1969); M. Tinkham, Introduction to Superconductivity (McGrawHill, New York, 1996).

[9] Yu. V. Nazarov, Phys. Rev. Lett. 73, 1420 (1994); Yu. V. Nazarov, Superlattices Microstruct. 25, 1221 (1999).

[10] A. Millis et al., Phys. Rev. B 38, 4504 (1988); M. Fogelstrom, Phys. Rev. B 62, 11812 (2000); J. C. Cuevas et al., Phys. Rev. B 64, 104502 (2001).

[11] D. Huertas-Hernando et al. (unpublished).

[12] J. Rammer and H. Smith, Rev. Mod. Phys. 58, 323 (1986).

[13] T. Tokuyasu et al., Phys. Rev. B 38, 8823 (1988).

[14] K. Xia et al., cond-mat/0107589.

[15] W. J. Gallagher et al., Phys. Rev. B 21, 962 (1980); R. Meservey and P. M. Tedrow, Phys. Rep. 238, 173 (1994).

[16] In particular, we found that the parameters that control the injection of the "ferromagnetic" and "superconducting" correlations are inversally proportional to the average time that it takes for the quasiparticles to probe each of the junctions that connect the node with the reservoirs $\left(\tau_{\mathrm{S}}=\right.$ $\hbar / \tilde{\Delta}$ and $\tau_{\mathrm{F}}=\hbar / \tilde{h}$, respectively).

[17] There is also a term $I_{\mathrm{J}}$ in $I_{\mathrm{e}}$, which describes supercurrent between the two nodes: $I_{\mathrm{J}}(t)=I_{\mathrm{J}}(\mathrm{eV}) \sin (2 \mathrm{eVt}+\Phi)$. For a dc bias voltage, this Josephson current alternates and does not contribute to dc electric current. 